\title{
Frecuencia, Severidad y Ubicación de Erupciones Acneiformes en Hombres y Mujeres tras una Cirugía Ortognática. Estudio Descriptivo Longitudinal
}

\author{
Frequency, Severity and Location of Acneiform Eruptions in Men \\ and Women after Orthognathic Surgery. Longitudinal Descriptive Study
}

Gracia Abuter, Benjamín'; Noguera Pantoja, Alfredo²; Sat Yaber, María Ignacia'; Carranza Morales, Francisca²; Ramírez Lobos, Valeria ${ }^{3}$ \& Solé Ventura, Pedro ${ }^{4}$

GRACIA, A. B.; NOGUERA, P. A.; SAT, Y. M. I.; CARRANZA, M. F.; RAMíREZ, L. V. \& SOLÉ, V. P. Frecuencia, severidad y ubicación de erupciones acneiformes en hombres y mujeres tras una cirugía ortognática. Estudio descriptivo longitudinal. Int. J. Odontostomat., 11(2):217-223, 2017.

RESUMEN: Describir la frecuencia de erupciones acneiformes y/o exacerbaciones de un acné previo tras una cirugía ortognática. La muestra consta de 57 pacientes $(n=57)$ de ambos sexos, sometidos a una cirugía ortognática, los cuales fueron evaluados en: el preoperatorio (0-7 días previos); en distintas etapas de la cirugía; postoperatorio inmediato ( 7 - 15 días post cirugía) y postoperatorio mediato ( $30-40$ días postquirúrgicos). En todos los controles clínicos mencionados se determinó la presencia/ausencia, ubicación, severidad y diagnóstico de las erupciones acneiformes. El 52,6\% de los pacientes sometidos a cirugía ortognática presentaron erupciones acneiformes, siendo mayores en las mujeres en comparación con los hombres. La severidad de las erupciones acneiformes es mayor en el postoperatorio inmediato en comparación al preoperatorio y postoperatorio mediato. La ubicación más frecuente del acné corresponde a la región frontal, tanto en el preoperatorio $(22,8 \%)$ como en el postoperatorio inmediato $(31,6 \%)$. En el postoperatorio mediato la zona más frecuente es la geniana $(39 \%)$. La frecuencia de acné post cirugía ortognática es elevada, siendo mayor en mujeres que en hombres. La severidad de este acné es mayor en el postoperatorio inmediato. La región frontal corresponde a la zona más frecuente de aparición de las erupciones acneiformes en el postoperatorio inmediato y la zona geniana en el postoperatorio mediato. El diagnóstico de estas erupciones acneiformes corresponde a un acné esteroidal, por lo que se puede sugerir un posible plan de tratamiento, con el fin de mejorar el postoperatorio de las pacientes y evitar, en lo posible, futuras manifestaciones en nuevas pacientes sometidas a este tipo de cirugía.

PALABRAS CLAVES: Acné; Cirugía ortognática; Estrés postquirúrgico.

\section{INTRODUCCIÓN}

La cirugía ortognática es una intervención quirúrgica realizada para corregir las asimetrías maxilomandibulares. Con dicha cirugía, se generan una serie de movimientos de los tejidos duros y blandos maxilofaciales utilizando patrones de armonía, estética y funcionalidad (López Rodríguez et al., 2004). La cirugía ortognática permite lograr una adecuada función masticatoria, fonética y respiratoria, mejorando así, la calidad de vida de los individuos afectados por estas desarmonías dentomaxilares. El aumento progresivo del nivel cultural y económico de nuestra sociedad, sumado a los avances de la tecnología de hoy en día, explican el hecho de que cada vez se realicen con mayor frecuencia este tipo de tratamientos ortodóncico - quirúrgicos, con bajos índices de complicaciones reportadas a corto y largo plazo (Alves e Silva et al., 2013).

\footnotetext{
${ }^{1}$ Cirujano Dentista, Universidad de los Andes, Chile.

${ }^{2}$ Cirujano Dentista, Residente Cirugía Bucal y Maxilofacial. Universidad de los Andes, Chile.

${ }^{3}$ Magíster Salud Pública, Universidad de los Andes, Chile.

${ }^{4}$ Cirujano Maxilofacial. Universidad de los Andes, Chile.
} 
GRACIA, A. B.; NOGUERA, P. A.; SAT, Y. M. I.; CARRANZA, M. F.; RAMíREZ, L. V. \& SOLÉ, V. P. Frecuencia, severidad y ubicación de erupciones acneiformes en hombres y mujeres tras una cirugía ortognática. Estudio descriptivo longitudinal. Int. J. Odontostomat.,11(2):217-223, 2017.

Una serie de fármacos son utilizados con fines analgésicos y antiinflamatorios en estos pacientes durante y posterior a la cirugía, dentro de los cuales encontramos a los corticoides. Dentro de los efectos no deseados de la administración de este tipo de fármacos cabe destacar la presencia de alteraciones dermatológicas (Rallis et al., 2004; Chegini \& Dhariwal, 2012).

Se han descrito en la literatura la aparición de erupciones acneiformes en pacientes intervenidos de cirugía ortognática con dos diagnósticos dermatológicos y de distinta incidencia. El autor Rallis, determinó que este cuadro dermatológico corresponde a dermatitis perioral, la cual se manifestó en 6 mujeres adultas de un total de 63 sometidas a este tipo de cirugía (Rallis et al.). Por otro lado, el autor Precious, determinó que las erupciones acneiformes manifestadas tras la cirugía ortognática, correspondían a acné esteroidal, observado en 8 mujeres de un total 1.276 pacientes adultos. En ambos estudios, las erupciones acneiformes aparecieron exclusivamente en pacientes de género femenino (Precious et al., 1992).

La relevancia de la aparición de estas erupciones acneiformes en pacientes sometidos a cirugía ortognática, radica en la incomodidad del punto de vista estético y la posibilidad de la aparición de secuelas permanentes en la piel de los pacientes tratados. Se deben considerar los efectos sociales y psicológicos que genera la presencia de acné en los pacientes, por ende, es de suma importancia identificar este efecto no deseado y entregarle un tratamiento que permita su resolución. Gupta \& Gupta (1998) destacan la importancia de reconocer signos y síntomas de depresión en pacientes con patologías dermatológicas.

La explicación de por qué este cuadro dermatológico tiene predilección por el género femenino, podría deberse a la interacción entre los factores hormonales, el estrés quirúrgico y el uso de corticoides administrados por vía parenteral. Sin embargo, al desconocer su etiología, es relevante determinar la prevalencia, etiología y diagnóstico de este cuadro para poder plantear una solución, por lo que esto es lo que motiva el desarrollo del presente trabajo de investigación.

El objetivo general del presente estudio corresponde a determinar la frecuencia, severidad, ubicación y diagnóstico de las erupciones acneiformes con y sin acné previo en sujetos sometidos a una cirugía ortognática.

\section{MATERIAL Y MÉTODO}

Este estudio corresponde a un estudio descriptivo longitudinal con un tiempo de seguimiento de 60 días. Esta investigación fue aprobada por los Comités de Investigación y Subcomité de Ética de la Facultad de Odontología de la Universidad de los Andes, Santiago de Chile.

Participantes: La población del estudio se distribuye en Chile, incluyendo a pacientes provenientes de: II región de Antofagasta, región Metropolitana, $\mathrm{V}$ región de Valparaíso y VI región del Libertador General Bernardo O'Higgins. Los pacientes fueron sometidos a una cirugía ortognática; Osteotomía maxilar tipo Le Fort I, osteotomía sagital de rama mandibular bilateral (OSRMB), genioplastía y/o combinaciones de estas, a cargo del mismo cirujano maxilofacial, bajo anestesia general y local. Las cirugías se llevaron a cabo entre diciembre del 2012 a Julio del 2014, en clínicas particulares y hospitales públicos de la región Metropolitana, Santiago de Chile. El manejo farmacológico de estos pacientes durante su hospitalización incluyó la administración de antibióticos (cefazolina 1gr cada $8 \mathrm{hrs}$ EV), antiinflamatorios no esteroidales (AINEs) (ketoprofeno $100 \mathrm{mg}$ cada $12 \mathrm{hrs} \mathrm{EV}$, ketorolaco 30 mg cada 8 hrs EV) y corticoides (dexametasona $4 \mathrm{mg}$ cada 8 hrs EV) durante 3 a 4 días.

Los criterios de inclusión incluyen todos aquellos pacientes sometidos a una cirugía ortognática; Osteotomía maxilar tipo Le Fort I, osteotomía sagital de rama mandibular bilateral (OSRMB), genioplastía y/o combinaciones de estas realizadas; pacientes que recibieron tratamiento con dexametasona (4 mg cada 8 hrs EV) durante su hospitalización ( 3 a 4 días) y pacientes que presentaron o no acné en el examen pre operatorio. Dentro de los criterios de exclusión se consideró pacientes que presentaron alguna enfermedad sistémica.

Obtención de datos: Los pacientes accedieron de manera voluntaria a participar del estudio firmando un consentimiento informado. Cada paciente se evaluó a través de un examen clínico preoperatorio ( 0 - 7 días previos a la cirugía), en el postoperatorio inmediato ( 7 a 15 días tras la cirugía) y postoperatorio mediato (30 a 40 días tras la cirugía), confeccionándose un instrumento de registro de esta información. El registro incluyó datos personales, antecedentes sistémicos y antecedentes dermatológicos. Además, se realizó un examen clínico pre y postoperatorio en donde se de- 
terminó la presencia/ausencia, ubicación y severidad de las erupciones acneiforme. Los operadores correspondieron a dos estudiantes de sexto año de Odontología de la Universidad de los Andes, quienes fueron calibrados por una dermatóloga en donde se definió la presencia de acné como una erupción cutánea, papular o pápulo -pustular, con un tamaño desde 3 $\mathrm{mm}$ a $1 \mathrm{~cm}$. de diámetro, de color rosado a rojo. La ubicación del acné se determinó en las siguientes regiones: frontal, nasal, geniana, perioral, mentoniana, hombros, tórax y espalda. En caso de presentar acné, se clasificó con la escala de severidad propuesta por Hayashi et al. (2008). Si es que el acné involucraba más de una zona, se eligió la zona más severa para la clasificación. Tanto en el postoperatorio inmediato como mediato se realizó un examen clínico en búsqueda de erupciones acneiformes, valorando ubicación y severidad, realizado por el mismo operador que realizó el examen preoperatorio.

Análisis Estadístico: El análisis estadístico se realizó con el software Stata versión 12. Las variables cuantitativas se presentaron con sus medianas y rangos intercuartílicos ya que no presentaron una distribución gaussiana; las variables categóricas se presentaron a través de frecuencias y porcentajes. Todo el análisis se realizó estratificado por género y en los distintos tiempos: preoperatorio, postoperatorio inmediato y postoperatorio mediato.

\section{RESULTADOS}

De una muestra inicial de 63 pacientes $(n=63)$, 6 pacientes de género femenino fueron excluidos del estudio por presentar enfermedades sistémicas (epilepsia, hipertensión arterial, resistencia a la insulina, trastorno de secreción plaquetaria e hipotiroidismo).
No hubo abandono del estudio por parte de los pacientes durante los exámenes postoperatorios. Por lo tanto, la muestra final incluyó a 57 pacientes $(n=57)$, conformada por 45 pacientes de género femenino y 12 de género masculino, donde todos los participantes finalizaron los exámenes postoperatorios propuestos, lo que se esquematiza diagrama de flujo (Fig. 1). Los datos demográficos y clínicos de los pacientes se presentan en la Tabla I.

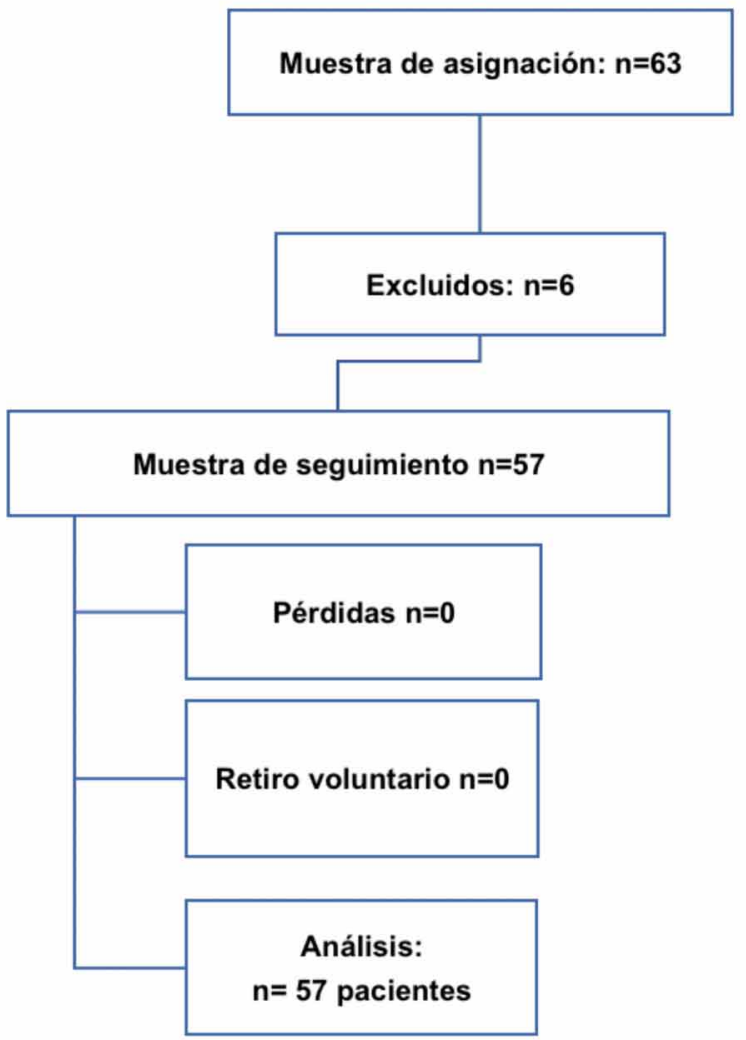

Fig.1. Diagrama de Flujo de participantes del estudio.

Tabla I. Características de los participantes.

\begin{tabular}{|c|c|c|}
\hline \multicolumn{3}{|c|}{ Características de los participantes } \\
\hline Sexo & Femenino $n=45(79 \%)$ & Masculino $n=12(21 \%)$ \\
\hline Edad & \multicolumn{2}{|c|}{ Mediana $=23$ años (Rango intercuartílico de 16 a 47 años) } \\
\hline \multirow{2}{*}{ Antecedentes previos de acné } & No: $n=14(73,7 \%)$ & No: $n=5(26,3 \%)$ \\
\hline & $\mathrm{Si}: \mathrm{n}=31(81,6 \%)$ & $\mathrm{Si}: \mathrm{n}=7(18,4 \%)$ \\
\hline \multirow{2}{*}{ Tratamiento dermatológico recibido } & No: $n=24(53,3 \%)$ & No: $n=9(75 \%)$ \\
\hline & Si : n= $21(46,6 \%)$ & $\mathrm{Si}: \mathrm{n}=3(25 \%)$ \\
\hline \multirow{2}{*}{ Tipo de tratamiento } & Tópico: n= 11 (50\%) & Tópico: n= $1(33,3$ \%) \\
\hline & Farmaœológico: $n=11(50 \%)$ & Farmacológico: $n=2(66,7 \%)$ \\
\hline
\end{tabular}


GRACIA, A. B.; NOGUERA, P. A.; SAT, Y. M. I.; CARRANZA, M. F.; RAMÍREZ, L. V. \& SOLÉ, V. P. Frecuencia, severidad y ubicación de erupciones acneiformes en hombres y mujeres tras una cirugía ortognática. Estudio descriptivo longitudinal. Int. J. Odontostomat., 11(2):217-223, 2017.

Tabla II. Frecuencia de acné en la muestra total.

\begin{tabular}{lcccc}
\hline & \multicolumn{2}{c}{ Preoperatorio } & \multicolumn{2}{c}{ Postoperatorio } \\
\cline { 2 - 5 } & $\mathrm{n}$ & $\%$ & $\mathrm{n}$ & $\%$ \\
\cline { 2 - 5 } Con acné & 19 & 33,3 & 30 & 52,6 \\
Sin acné & 38 & 66,7 & 27 & 47,4 \\
Total & 57 & 100 & 57 & 100 \\
\hline
\end{tabular}

Frecuencia del Acné . Frecuencia del acné post cirugía en la muestra total: Para analizar la variable acné post cirugía, fue necesario agrupar dentro de esta a las variables acné en el examen post operatorio inmediato y acné en el examen postoperatorio mediato. En la tabla 2 se muestra la frecuencia de acné en los pacientes hombres y mujeres. Durante el examen preoperatorio, 19 pacientes $(33,3 \%)$ de la muestra total de participantes presentaban acné previo a la cirugía. Después de la cirugía ortognática, 30 pacientes $(52,6 \%)$ presentaron acné.

De un total de 12 participantes de género masculino, 2 pacientes $(16,7 \%)$ presentaron acné previo a la cirugía. Después de la cirugía, 3 pacientes presentaron acné $(25 \%)$. En el caso de las participantes mujeres, de un total de 45 participantes de género femenino, 17 pacientes $(37,8 \%)$ presentaron acné previo a la cirugía. Después de la cirugía, 27 pacientes de género femenino (60\%) presentaron acné.

Severidad del Acné postquirúrgico: En el examen preoperatorio, la tabla 3 muestra que, de un total de 57 participantes del estudio, $19(33,3 \%)$ pacientes

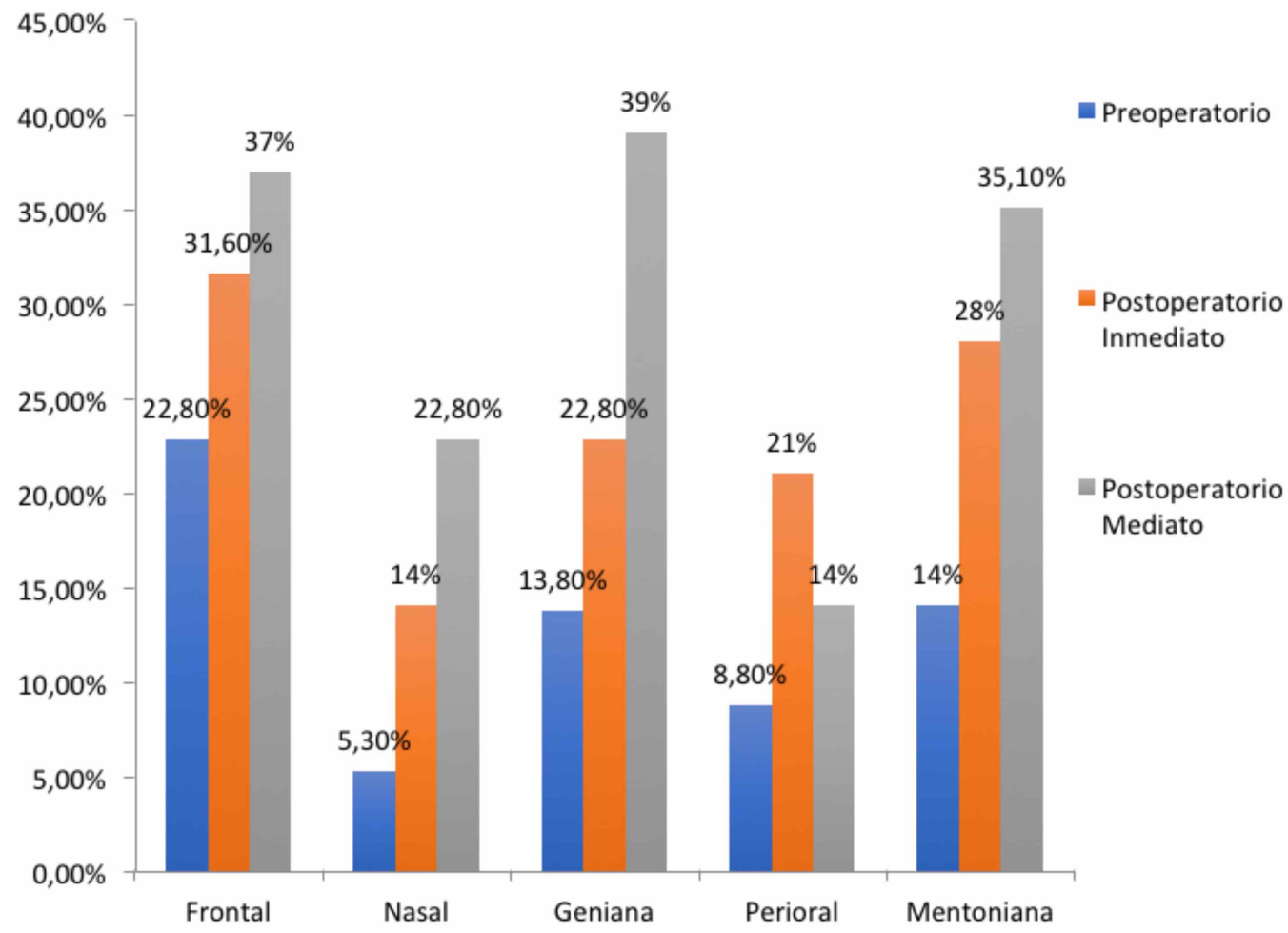

Fig. 2. Ubicación del acné preoperatorio, postoperatorio inmediato y mediato en la región facial

Tabla III. Severidad del acné.

\begin{tabular}{lcccccc}
\hline Severidad & Preoperatorio & \multicolumn{2}{c}{$\begin{array}{c}\text { Postoperatorio } \\
\text { Inmediato }\end{array}$} & \multicolumn{2}{c}{$\begin{array}{c}\text { Postoperatorio } \\
\text { Mediato }\end{array}$} \\
& $\mathrm{n}$ & $\%$ & $\mathrm{n}$ & $\%$ & $\mathrm{n}$ & $\%$ \\
\hline Leve & 15 & 79 & 13 & 43,30 & 17 & 53,10 \\
Moderado & 3 & 15,80 & 14 & 46,70 & 12 & 37,50 \\
Moderado - Severo & 1 & 5,30 & 3 & 10 & 3 & 9,40 \\
Severo & 0 & 0 & 0 & 0 & 0 & 0 \\
Total & 19 & 100 & 30 & 100 & 32 & 100 \\
\hline
\end{tabular}


presentaron acné. De estos 19 pacientes, $15(79 \%)$ de ellos presentaron acné leve, $3(15,8 \%)$ pacientes presentaron acné moderado y $1(5,3 \%)$ paciente presentó acné moderado - severo. En el examen postoperatorio inmediato, la tabla 3 muestra que, de un total de 57 participantes del estudio, 30 (52,6\%) pacientes presentaron acné en el examen postoperatorio inmediato. De estos 30 pacientes, 13 $(43,3 \%)$ de ellos presentaron acné leve, 14 (46,7\%) de ellos presentaron acné moderado y $3(10 \%)$ pacientes presentaron acné moderado severo. Finalmente, en el postoperatorio mediato la tabla 3 muestra que, de un total de 57 participantes del estudio, 32 (56,1 $\%)$ pacientes presentaron acné en el examen postoperatorio mediato. De estos 32 pacientes, 17 $(53,1 \%)$ de ellos presentaron acné leve, $12(37,5 \%)$ de ellos presentaron acné moderado y $3(9,4 \%)$ pacientes presentaron un cuadro de acné moderado severo.

Ubicación del Acné: La Figura 2 muestra la distribución de la ubicación del acné en un total de 57 pacientes; en el preoperatorio, postoperatorio inmediato y mediato. Durante el preoperatorio, el acné comprometió en mayor proporción las regiones: frontal $(22,8 \%)$ y geniana $(15,8 \%)$. Durante el postoperatorio inmediato, el acné post cirugía comprometió en mayor proporción las regiones: frontal $(31,6 \%)$, mentoniana $(28 \%)$ y geniana (22 \%). Durante el postoperatorio mediato, el acné post cirugía comprometió en mayor proporción a la región geniana (39 \%). En segundo y tercer lugar, las regiones más comprometidas fueron las regiones: frontal $(37 \%)$ y mentoniana $(35 \%)$.

\section{DISCUSIÓN}

Frecuencia del acné: Este estudio muestra que la frecuencia de las erupciones acneiformes y/o exacerbaciones de un acné previo aumentó una vez realizada la cirugía. Estos resultados, difieren con aquellos obtenidos por Precious y Rallis, donde las frecuencias de acné esteroidal y de dermatitis perioral fueron menores en comparación con las obtenidas en el presente estudio. Esta diferencia se puede atribuir a que en los estudios anteriores no fueron incluidos pacientes que presentaran acné durante el preoperatorio, por lo tanto, sólo se midió la aparición de la erupción acneiforme, y no la exacerbación del acné previo. Es importante mencionar que los estudios realizados por Precious y Rallis se realizaron en pacientes de nacionalidad griega, en el caso de Rallis, y canadiense, en el caso de Precious; lo cual también podría explicar la diferencia en la frecuencia de la erupción acneiforme en relación al estudio actual (Rallis et al.; Precious et al.).

Frecuencia de acné según texto: El acné post cirugía fue manifestado en ambos géneros, sin embargo, su frecuencia fue mayor en el género femenino. Por lo tanto, los resultados difieren con los resultados obtenidos por Precious y Rallis, donde tanto el acné esteroidal como la dermatitis perioral, fueron manifestados únicamente en mujeres (Rallis et al.; Precious et al.). Los resultados del presente estudio coinciden con aquellos obtenidos por Cunliffe \& Gould (1979), donde se determinó que existe una mayor prevalencia de acné en el género femenino. Este se atribuye al uso de: cosméticos, anticonceptivos orales de predominancia androgénica y a la dieta. A pesar de que en el estudio antes mencionado se midió la prevalencia del acné vulgar, se puede realizar una analogía entre éste y la erupción acneiforme tras la cirugía ortognática debido a su similitud en términos de etiopatogenia (Cunliffe \& Gould).

Severidad del acné: En el examen preoperatorio y postoperatorio mediato, la mayoría del acné post cirugía se presentó en su forma leve; a diferencia del examen postoperatorio inmediato, donde la mayoría del acné post cirugía se presentó en su forma moderada. Este hecho puede deberse a que la erupción acneiforme que se manifiesta en el postoperatorio inmediato puede atribuirse tanto a la cirugía ortognática, como al estrés y corticoides (Hill \& Walker, 2001). Cabe destacar que, a pesar de que la frecuencia de erupciones acneiformes tras la cirugía fue elevada, la mayoría de estas manifestaciones fueron clasificadas como formas leves. Sin embargo no se puede comparar estos resultados con las publicaciones realizadas por Precious y Rallis, ya que no consideraron la severidad de las erupciones acneiformes; sino que sólo midieron la presencia o ausencia de estas (Rallis et al.; Precious et al.). Actualmente, no existe consenso acerca de una escala objetiva que permita clasificar la severidad del acné ya que la mayoría de las clasificaciones están basadas en opiniones de expertos y no en estudios conducidos para confirmar estas clasificaciones. En este estudio, se utilizó la escala de severidad creada por Hayashi. Esta escala es creada por este grupo de investigadores realizando concordancia entre: comparaciones realizadas por dermatólogos expertos, fotografías y conteo de lesiones inflamatorias. Este método, basado en métodos científicos, se presenta como uno de los más confiable y 
útil en la estimación de la severidad del acné ya que se basa en el número de lesiones y no en el tipo de éstas (Hayashi et al.).

Ubicación del acné: La región facial que se vio mayormente afectada por la aparición de las erupciones acneiforme en el preoperatorio y en el postoperatorio inmediato fue la región frontal; sin embargo, en el postoperatorio mediato la zona que presentó mayor frecuencia fue la región geniana. Sin embargo, actualmente no existen otras publicaciones con las que se pueda comparar este resultado ya que en los estudios realizados por Precious y Rallis no se determinó la ubicación del cuadro dermatológico en la región facial. En una submuestra del estudio se decidió evaluar la ubicación del acné en otras regiones del cuerpo: hombro, espalda y tórax. Cabe destacar que no se manifestó la patología en la espalda. Las zonas comprometidas por el acné postcirugía tienen gran relevancia, ya que coinciden con el diagnóstico de acné esteroidal. Según lo observado en el estudio de Precious et al. en el cual las erupciones cutáneas afectaron la región de la cara, parte superior del tronco y extremidades superiores. En ese se atribuyó que la causa de la aparición del acné esteroidal es la administración de corticoides parenterales en un corto periodo de tiempo. Por lo tanto, se infiere que son estos fármacos los responsables de las manifestaciones dermatológicas (Precious et al.).

Etiología de la erupción acneiforme: Rallis et al. postulan que dentro de los múltiples factores etiológicos de esta erupción acneiforme se encuentran: estrés emocional, uso de fijación interna rígida y la incapacidad para contener la saliva causada por la hipoestesia tras una cirugía ortognática (Rallis et al.). No se ha reportado este tipo de reacción dermatológica en otro tipo de cirugía maxilofacial donde también son utilizados corticoides en el postoperatorio. Por lo tanto, se excluye como factor exclusivo causal sólo el uso de estos fármacos, sino más bien una etiología multifactorial donde participan estos fármacos. Sin embargo, si se ha reportado un aumento del cortisol, producto del estrés generado en cirugía de terceros molares incluidos (Hill \& Walker).

\section{CONCLUSIONES}

La frecuencia de erupciones acneiformes fue de un $52,6 \%$ de los pacientes de los sometidos a una cirugía ortognática. La frecuencia de los pacientes de género femenino que desarrollaron el cuadro fue mayor que los de género masculino. La severidad de las erupciones acneiformes en el examen preoperatorio y postoperatorio mediato se presentó en forma leve; a diferencia del examen postoperatorio inmediato, donde la mayoría del acné se presentó en forma moderada. La ubicación en la región facial que se vio mayormente afectada por la aparición de las erupciones acneiforme en el preoperatorio y en el postoperatorio inmediato fue la región frontal; Sin embargo, en el postoperatorio mediato la zona que presentó mayor frecuencia fue la región geniana. Podría plantearse la existencia de una relación entre el ciclo menstrual, estrés quirúrgico y niveles de cortisol aumentados con la aparición de acné post-quirúrgico, pero falta evidencia que avale esta relación. Es muy importante esta observación ya que aquí podría encontrarse la explicación de por qué afecta más a mujeres.

\section{AGRADECIMIENTOS}

Los autores agradecen a la Dra. María Luisa Saenz de la Clínica Santa María por su aporte en la realización de este trabajo de investigación.

GRACIA, A. B.; NOGUERA, P. A.; SAT, Y. M. I.; CARRANZA, M. F.; RAMÍREZ, L. V. \& SOLÉ, V. P. Frequency, severity and location of acneiform eruptions in men and women after orthognathic surgery. longitudinal descriptive study. Int. J. Odontostomat., 11(2):217-223, 2017.

ABSTRACT: Describe the frequency of acneiform eruptions and / or exacerbations of a previous acne after orthognathic surgery. The sample consisted of 57 patients $(n=57)$ of both genders, undergoing orthognathic surgery, who were evaluated with a follow-up of 2 postoperative months, at different stages of surgery; Preoperative (0-7 days), immediate postoperative (7-15 days) and mediate postoperative (30-40 days). The presence / absence, location, severity and diagnosis of acneiform eruptions were determined in all clinical controls. The frequency of acneiform eruptions corresponds to $52.6 \%$ of patients undergoing orthognathic surgery, being higher in women compared to men in relation to the presence of acneiform eruptions and / or exacerbations of a previous acne after the intervention. The severity of acneiform eruptions is greater in the immediate postoperative period compared to the preoperative and mediate postoperative period. The most frequent location to be found in the facial region is in the frontal area, both in the preoperative $(22.8 \%)$ and in the immediate postoperative period (31.6\%). In the postoperative period, the most frequent is the genial area (39\%). The appearance of acneiform 
eruptions corresponds to steroidal acne. The frequency of acne post orthognathic surgery is high, being higher in women than in men. The severity of this acne is greater in the immediate postoperative period. The frontal region corresponds to the most frequent area of onset of acneiform eruptions in the immediate postoperative period and the genial area in the postoperative period. The diagnosis of these acneiform eruptions corresponds to a steroidal acne, so it is possible to suggest a possible treatment plan, in order to improve the postoperative of the patients and to avoid, as far as possible, future manifestations in new patients undergoing this type of surgery.

KEY WORD: Acne; Orthognathic surgery; Postsurgical stress.

\section{REFERENCIAS BIBLIOGRÁFICAS}

Alves e Silva, A. C.; Carvalho, R. A. S.; Santos, T. de S.; Rocha, N. S.; Gomes, A. C. A. \& de Oliveira e Silva, E. D. Evaluation of life quality of patients submitted to orthognathic surgery. Dent. Press J. Orthod., 18(5):107-14, 2013.

Chegini, S. \& Dhariwal, D. K. Review of evidence for the use of steroids in orthognathic surgery. Br. J. Oral Maxillofac. Surg., 50(2):97-101, 2012.

Cunliffe, W. J. \& Gould, D. J. Prevalence of facial acne vulgaris in late adolescence and in adults. Br. Med. J., 1(6171):1109-10, 1979

Gupta, M. A. \& Gupta, A. K. Depression and suicidal ideation in dermatology patients with acne, alopecia areata, atopic dermatitis and psoriasis. Br. J. Dermatol., 139(5):846-50, 1998.

Hayashi, N.; Akamatsu, H.; Kawashima, M \& Acne Study Group. Establishment of grading criteria for acne severity. J. Dermatol., 35(5):255-60, 2008.

Hill, C. M. \& Walker, R. V. Salivary cortisol determinations and selfrating scales in the assessment of stress in patients undergoing the extraction of wisdom teeth. Br. Dent. J., 191(9):513-5, 2001.

López Rodríguez, A.; Soto Fernández, A.; Sarracent Pérez, H.; Pérez Varela, H.; Pantoja Valdés, D. \& Muñiz Manzano, E. Cirugía ortognática: un medio para adquirir belleza y salud. Rev. Cuba. Estomatol., 41(2), 2004.

Precious, D. S.; Hoffman, C. D. \& Miller, R. Steroid acne after orthognathic surgery. Oral Surg. Oral Med. Oral Pathol., 74(3):279-81, 1992

Rallis, G.; Papakosta, V.; Petrakogiannis, C. \& Zachariades, N. Perioral dermatitis following orthognathic surgery. Plast. Reconstr. Surg., 113(2):771-3, 2004.
Dirección para correspondencia:

Dr. Benjamín Gracia

Universidad de los Andes

Facultad de Odontología

Monseñor Álvaro del Portillo No 12.455

Las Condes

Santiago

CHILE

E-mail: drpedrosole@gmail.com

Recibido : 13-03-2017

Aceptado: 05-06-2017 